\title{
Entomofauna polinizadora de Eucalyptus nitens en huertos semilleros del centro sur de Chile
}

\section{Pollinator insects of Eucalyptus nitens in a clonal seed orchard in South Central Chile}

\author{
María-José Sanzana a*, Luis E Parra ${ }^{\text {a }}$, Hugo A Benítez ${ }^{\text {a,b }}$, Jaime Espejo c \\ *Autor de correspondencia: ${ }^{a}$ Universidad de Concepción, Facultad de Ciencias Naturales y Oceanográficas, Departamento de \\ Zoología, casilla 160-C, Concepción, Chile, tel.: (41)2203414, mariajosanzana@udec.cl

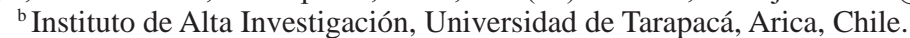 \\ ${ }^{c}$ Universidad de Concepción, Programa Postgrado Ciencias Forestales, Concepción, Chile.
}

\begin{abstract}
SUMMARY
Variations can be observed in pollinator assemblages of exotic species in response to changes in environment or to variables that did not receive much attention. This paper is the first approximation of the abundance of pollinator species associated with Eucalyptus nitens in a Clonal seed-bank plantation (CSBP) of the farm "Rucamanqui", Huepil, Biobío Region in Chile. Between October and November 2010, we examined the variation of pollinator's entomofauna of E. nitens. Environmental factors, such as temperature and the number of open flowers per plant, were registered to determine the level of dependence on the frequency of visits. The most abundant insects visiting E. nitens belonged to the orders Coleoptera, Hymenoptera, Diptera and Lepidoptera. However, the proportion of individuals in each group of flower visitors varied considerably among the sampling months: Hymenoptera (203), Coleoptera (21) in October and Coleoptera (280), Hymenoptera (57) in November. For Hymenoptera, environmental temperature was significant compared to frequency of visitors; whereas for Coleoptera, the number of open flowers per plant has been significant for insect activity. Results show that the visit frequency of pollinators in E. nitens is highly variable within the sampling period and temperature and density of open flowers per plant can produce variations within the group of pollinators.
\end{abstract}

Key words: Hymenoptera, Coleoptera, Diptera, abundance, temperature.

\section{RESUMEN}

Las variaciones que se pueden observar en los ensambles de polinizadores de especies exóticas, en respuesta a cambios en las variables ambientales o florales, no han recibido mucha atención. Este trabajo constituye la primera aproximación sobre la abundancia de especies polinizadoras, asociadas al Eucalyptus nitens en un huerto semillero clonal de la hacienda Rucamanqui, localidad de Huépil $\left(37^{\circ} 15^{\prime} \mathrm{S}\right.$ y $\left.71^{\circ} 55^{\prime} \mathrm{O}\right)$, región del Biobío. Entre octubre y noviembre de 2010, se examinó la variación de la entomofauna polinizadora de E. nitens. Se registraron además factores como la temperatura ambiental y el número de flores abiertas por planta para determinar el nivel de dependencia de la frecuencia de visitas. Los insectos más abundantes que visitaron a E. nitens pertenecen a los órdenes Coleoptera, Hymenoptera, Diptera y Lepidoptera. Sin embargo, la proporción del total de los individuos visitantes florales en cada uno de los grupos varió notablemente entre los meses de muestreo: Hymenoptera (203), Coleoptera (21) en octubre, y Coleoptera (280), Hymenoptera (57) en noviembre. Para Hymenoptera la temperatura ambiental fue significativa frente a la frecuencia de visitas y para Coleoptera el número de flores abiertas por planta fue significativo frente a la actividad de los insectos. Los resultados muestran que sobre E. nitens la frecuencia de visita de polinizadores es altamente variable dentro del período de muestreo y que la temperatura y la densidad de flores abiertas por planta pueden generar variación dentro de cada grupo de polinizadores.

Palabras clave: Hymenoptera, Coleoptera, Diptera, abundancia, temperatura.

\section{INTRODUCCIÓN}

Los insectos que intervienen en el proceso de polinización biótica, han desarrollado diferentes estrategias de alimentación, las que muchas veces están estrechamente relacionadas al tipo de especie vegetal a polinizar. Estas adaptaciones permiten a los insectos usar a las plantas como fuente de alimento (néctar, polen o aceites florales) o como sustrato para su reproducción (construcción de nichos, oviposición, etc.) (Brown y Freitas 2002). El éxito reproductivo de las especies vegetales depende de las interacciones con los visitantes florales como los polinizadores (Speight et al. 1999). Cerca del $70 \%$ de las plantas con flores posee una dependencia directa de los insectos para su reproducción y propagación (Seastedt y Crossley 1984). Por ello, el interés en el fenómeno de la polinización es comprensible, debido a que es de vital importancia para la producción comercial de cualquier tipo de cultivo, determinando así, la obtención de diversos productos vegetales. 
En Chile los huertos semilleros de especies exóticas se han especializado en el estudio y la obtención de semillas mejoradas genéticamente. Diversos estudios indican que Eucalyptus nitens (Deane et Maiden) Maiden posee características que resultan ventajosas sobre otras especies de eucaliptos, tales como: mayor velocidad de crecimiento y resistencia al frío que E. globulus Labill. y E. regnans F. Muell.; mejor adaptación a zonas altas, frías y lluviosas; madera de color claro y moderadamente densa en comparación con otras especies (Lopetegui 2002, INFOR 2004). Estas características han permitido que E. nitens sea una especie de suma importancia para las condiciones edafoclimáticas del centro sur de Chile. La producción de semilla a partir de la selección de árboles plus y su establecimiento en huertos semilleros, es la vía más utilizada para asegurar el requerimiento de esta especie, tanto en vivero como en actividades silvícolas. Sin embargo, la viabilidad de sus poblaciones debe ser asegurada por la producción de semillas, lo que requiere necesariamente de la presencia de insectos polinizadores (Proctor et al. 1996). Son estos insectos los que contribuyen con casi el $80 \%$ de la polinización alógama o cruzada (Fernández 2010), en donde la flor requiere del polen de otra flor u otra planta. En Chile, antecedentes sobre visitantes florales de eucaliptos, y las fluctuaciones que puedan presentar estos insectos, son escasos (Lopetegui 2002, Fernández 2010).

Estudios recientes han sugerido que las interacciones planta-polinizador pueden ser menos especializadas de lo que se pensaba. Las causas que generan esta variación interpoblacional en el conjunto de polinizadores de una determinada especie vegetal son variadas. Muchos autores indican que la relación entre la densidad y frecuencia de visitas puede ser muy variable (Kearns y Inouye 1993). Según Morales y Aizen (2002), los trastornos en el hábitat pueden alterar las interacciones entre insectos polinizadores y plantas debido a la influencia de la composición $\mathrm{y}$ abundancia de flores. Estos autores comentan que este proceso es más evidente cuando el trastorno está asociado a la invasión de plantas exóticas, que representan una abundante fuente de alimento (néctar y polen) tanto para visitantes florales nativos como introducidos.

El otro conjunto de factores determinantes sobre las variaciones de polinizadores, son los factores abióticos. Entre ellos, las condiciones climáticas que imperan en el sitio donde se encuentran las plantas, son factores que pueden influir fuertemente sobre el comportamiento y la actividad de los polinizadores (Rozzi et al. 1997). Los polinizadores son especialmente sensibles a cambios de temperatura, velocidad del viento y nubosidad, condiciones claves que poseen una alta correlación con el éxito reproductivo de las plantas con flores, cobrando una marcada importancia bajo eventuales variaciones climáticas (Torres-Díaz et al. 2007). Sin embargo, las consecuencias que puedan tener estas variaciones climáticas sobre la tasa de visita de los polinizadores, han sido poco estudiadas. Todo lo anterior sugiere que las variaciones climáticas como la temperatura y los aspectos de la floración de cada especie de planta, podrían generar diferentes patrones de actividad de insectos polinizadores.

Es importante tener en cuenta que los patrones de variación en sistemas planta-polinizador a lo largo de gradientes ambientales han sido estudiados en una variedad de escalas espaciales y ecológicas. Los trabajos publicados incluyen ejemplos de búsqueda de patrones relacionados con variaciones de altitud (Malo y Baonza 2002), latitud (Ollerton y Cranmer 2002), insularidad (Ollerton y Cranmer 2002, Jordano et al. 2003), fragmentación y complejidad del paisaje (Tscharntke y Brandl 2004) y estadío sucesional luego de un disturbio (Potts et al. 2003). Al mismo tiempo, estos estudios reflejan la dificultad inherente a la obtención de este tipo de información, lo cual plantea un compromiso constante entre resolución espacial (de local a global), resolución taxonómica (de especies individuales a la comunidad entera) y esfuerzo de muestreo (de unos pocos días a años).

En Chile se reporta la introducción de E. nitens en variados huertos semilleros y se conoce de la presencia de algunos polinizadores, aunque no son muchos los estudios que se tienen al respecto en el país (Lopetegui 2002, Fernández 2010). Ante esta situación y los antecedentes planteados, surge la necesidad de realizar la presente investigación en huertos semilleros del centro sur de Chile. Para ello, se plantea como hipótesis que con el aumento en la temperatura ambiental y en el número de flores abiertas por planta, incrementaría la actividad polinizadora de los insectos, reflejándose en un aumento de visitantes florales. El objetivo de este estudio es conocer la entomofauna polinizadora y las características del ensamble de visitantes florales (abundancia de especies y número de flores visitadas). Asimismo, también se analizan aspectos de la floración y del ambiente, que podrían estar directamente relacionados con la actividad y características de los visitantes florales (número de flores abiertas por planta y temperatura ambiental del huerto).

\section{MÉTODOS}

Área de estudio. El estudio se efectuó en el huerto semillero de E. nitens (Angiospermae: Myrtaceae), de la hacienda Rucamanqui de Forestal Mininco S.A, (región del Biobío), localizada a los $37^{\circ} 15^{\prime} \mathrm{S}$ y $71^{\circ} 55^{\prime} \mathrm{O}$, a una altitud media 485 m s.n.m.

Condiciones del huerto. El manejo del huerto semillero constó de fertilización (NPK), aplicación de agroquími$\cos ^{1}$ para la prevención de Gonipterus scutellatus Gyll. (Coleoptera, Curculionidae) y de podas sucesivas.

\footnotetext{
${ }^{1}$ Actara: insecticida de amplio espectro (Tiametoxam: 3-(2-cloro-tiazol-5-ilmetil)5-metil-(1,3,5)oxadiazinan-4-iliden-nitroamina) y Confidor: insecticida sistémico selectivo (Imidacloprid:1-(6-cloro- 3-piridilmetil) -N-nitroimidazolidin -2-ylidene amina).
} 
Estrategia de observación. Se seleccionaron plantas en forma aleatoria con floración en más de dos ramas. Durante el período de floración se realizó las observaciones de polinizadores, siguiendo el procedimiento descrito en Utelli y Roy (2000). Este consistió en tres períodos de $30 \mathrm{mi}-$ nutos de observación con dos observadores por planta, lo que resulta en una total de tres horas de observación al día. Este procedimiento se repitió durante nueve días, de manera que en el huerto se completó un total de 54 periodos de observación. Las observaciones se realizaron en días mayormente despejados con temperaturas superiores a los $10{ }^{\circ} \mathrm{C}$. Posteriormente, se contabilizó el número de flores visitadas por cada polinizador y el número de visitas por planta observada. Con el fin de discriminar entre visitantes y polinizadores efectivos, se observó el comportamiento de los insectos en la flor, verificando si se hizo probable el contacto entre anteras y pistilos. Una vez finalizada la observación y registro del total de especies de polinizadores que interactuaban con las inflorescencias de E. nitens, se procedió a la captura mediante red entomológica, después fueron puestos en frascos con alcohol y rotulados, para su posterior identificación.

Condiciones microclimáticas. Con el propósito de comparar las condiciones térmicas imperantes en cada una de los meses de muestreo, fue registrada la temperatura del aire mediante un registrador de temperatura de memoria permanente COX Tracer, instalado en el huerto, entre las 08:00 y las 17:00 h, durante todos los días que se observó polinizadores. Esto permitió conocer las temperaturas medias y su variación a lo largo de los nueve días de observación (cuadro 1). En el huerto semillero de E. nitens, la temperatura promedio en octubre fue $1,9{ }^{\circ} \mathrm{C}$ menor que en noviembre (cuadro 1).

Número de flores abiertas por planta. Con el propósito de examinar los posibles efectos del número de flores abiertas por planta sobre la de visita de polinizadores, cada día que se muestreó polinizadores se contabilizó el número de flores abiertas de cada planta muestreada (cuadro 1). Para el caso de noviembre, el promedio de inflorescencias por planta fue el doble a lo contabilizado en octubre (cuadro 1).

Procesamiento de muestras. Los ejemplares recolectados fueron determinados hasta el nivel de especie con la ayuda de especialistas y de guías taxonómicas. Este material fue sometido a microscopía electrónica de barrido (MEB) (Laboratorio de Microscopía Electrónica de la Universidad de Concepción) para determinar la presencia o ausencia de polen en el cuerpo de los insectos. Los tipos de polen encontrados fueron determinados taxonómicamente con la ayuda de especialistas. Todos los ejemplares fueron revisados a través de una lupa estereoscópica Olympus TL2 y fueron depositados en el museo de Zoología de la Facultad de Ciencias Naturales y Oceanográficas de la Universidad de Concepción (UCCC-MZUC).

Métodos estadísticos. Para determinar la relación de las variables, se utilizó el coeficiente de correlación de Pearson (r) para datos paramétricos y Spearman (rs) para datos no paramétricos, que reflejaron el grado de relación lineal que existe entre cada orden de insectos con el número de flores abiertas por planta, y la temperatura ambiental. Se aplicó la prueba de $t$ de Student para determinar las diferencias significativas entre i) el número de flores abiertas por planta y ii) la temperatura ambiental, con la frecuencia de visitas, previa evaluación de normalidad y homogeneidad de varianzas. Se utilizó el programa estadístico PAST 2.0.

\section{RESULTADOS}

Abundancia y visitantes florales. Durante la realización de este estudio se registró la ocurrencia de 741 individuos de un total de 17 especies (cuadro 2) de insectos visitantes florales, pertenecientes a cuatro órdenes. De estas especies registradas, ocho fueron comunes a los dos meses de muestreo, nueve fueron exclusivas: cuatro para el mes de octubre y cinco para el mes de noviembre. La mayor abundancia ocurre en Coleoptera (301) e Hymenoptera (260), seguido de Diptera (179) y Lepidoptera (1) (figura 1).

Cuadro 1. Temperatura $\left({ }^{\circ} \mathrm{C}\right)$ media diaria y número de flores abiertas por planta de cada día muestreado en octubre y noviembre en el huerto semillero de E. nitens.

Daily average temperature $\left({ }^{\circ} \mathrm{C}\right)$ and number of open flowers per plant for each day sampled in October and November (clonal seed orchard of E. nitens).

\begin{tabular}{|c|c|c|c|c|c|c|c|}
\hline \multicolumn{4}{|c|}{ Temperatura $\left({ }^{\circ} \mathrm{C}\right)$} & \multicolumn{4}{|c|}{ Número de flores abiertas } \\
\hline $\begin{array}{l}\text { Día de } \\
\text { octubre }\end{array}$ & $\begin{array}{c}\text { Media } \\
\text { diaria }\end{array}$ & $\begin{array}{c}\text { Día de } \\
\text { noviembre }\end{array}$ & $\begin{array}{l}\text { Media } \\
\text { diaria }\end{array}$ & $\begin{array}{l}\text { Día de } \\
\text { octubre }\end{array}$ & $\begin{array}{l}\text { Media } \\
\text { diaria }\end{array}$ & $\begin{array}{c}\text { Día de } \\
\text { noviembre }\end{array}$ & $\begin{array}{l}\text { Media } \\
\text { diaria }\end{array}$ \\
\hline 1 & 24,7 & 5 & 28,2 & 1 & 50 & 5 & 148 \\
\hline 8 & 24,3 & 12 & 23,6 & 8 & 48 & 12 & 106 \\
\hline 15 & 20,6 & 19 & 23,8 & 15 & 52 & 19 & 110 \\
\hline 22 & 17,1 & 26 & 16,3 & 22 & 59 & 26 & 123 \\
\hline 29 & 17,3 & - & - & 29 & 87 & - & - \\
\hline Promedio & 21,0 & - & 22,9 & Promedio & 59 & - & 122 \\
\hline
\end{tabular}


Cuadro 2. Insectos visitantes de E. nitens registrados entre octubre y noviembre de 2010, en el huerto semillero de la hacienda Rucamanqui, Huepil, región del Biobío.

Insect visitors to E. nitens reported between October and November 2010, in the clonal seed orchard of farm "Rucamanqui", Huepil, Biobío Region. * Species observed but not collected.

\begin{tabular}{|c|c|c|c|c|c|}
\hline Orden & Familia & Género & Especie & $\begin{array}{l}\text { Número de visitas } \\
\text { (\% de visitas) }\end{array}$ & Mes de ocurrencia \\
\hline \multirow[t]{7}{*}{ Hymenoptera } & Halictidae & Caenohalictus & C. thauca (Schrottky, 1909) & $118(15,77)$ & octubre, noviembre \\
\hline & & Caenohalictus & C. opacus (Friese, 1917) & $130(17,37)$ & octubre, noviembre \\
\hline & & Corynura & $s p$. & $1(0,13)$ & noviembre \\
\hline & Colletidae & Colletes & sp. & $3(0,40)$ & octubre \\
\hline & & Diphaglossa & $s p$. & $2(0,26)$ & noviembre \\
\hline & Apidae & Bombus & $s p$. & $4(0,53)$ & octubre, noviembre \\
\hline & & Apis & A. mellifera Linnaeus, 1758 & $2(0,26)$ & noviembre \\
\hline \multirow[t]{4}{*}{ Diptera } & Syrphidae & Syrphus & S. reedi Shannon, 1927 & $60(8,02)$ & octubre, noviembre \\
\hline & & Sterphus & S. coerulea (Rondani) & $43(5,74)$ & octubre, noviembre \\
\hline & Muscidae & No identificado & - & $42(5,61)$ & octubre, noviembre \\
\hline & & No identificado & - & $34(4,54)$ & octubre, noviembre \\
\hline \multirow[t]{5}{*}{ Coleoptera } & Peltidae & Decamerus & D. haemorroidalis Solier, 1849 & $43(5,74)$ & octubre, noviembre \\
\hline & Cerambycidae & Callideriphus & C. laetus Blanchard, 1851 & $2(0,26)$ & octubre \\
\hline & Melyridae & No identificado & - & $155(20,72)$ & noviembre \\
\hline & Mordellidae & Mordella & M. luctuosa Solier, 1851 & $100(13,36)$ & noviembre \\
\hline & Coccinellidae & * & - & $1(0,13)$ & octubre \\
\hline Lepidoptera & Pieridae & *Colias & C. vauthieri Guérin, 1829 & $1(0,13)$ & octubre \\
\hline
\end{tabular}

* Especies observadas pero no colectadas. -: Especie no identificada.

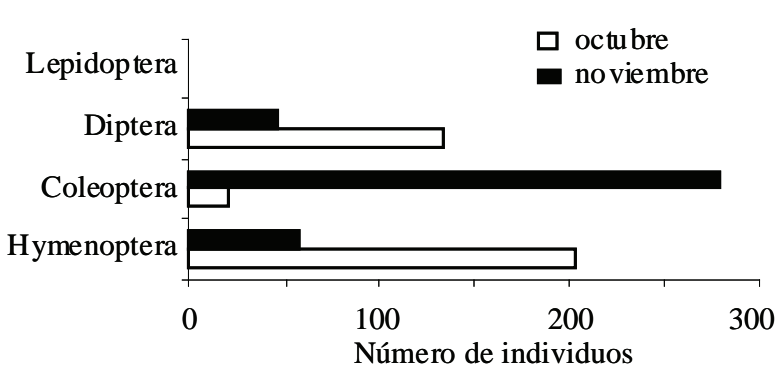

Figura 1. Representatividad de los taxones de insectos que visitaron a $E$. nitens recolectados en un período de octubre a noviembre de 2010, en el huerto semillero de la hacienda Rucamanqui, Huepil, región del Biobío.

Representativeness of the insect taxa that visited E. nitens collected from October to November 2010 in the clonal seed orchard of farm "Rucamanqui”, Huepil, Biobío Region.

La proporción del total de visitas florales en cada uno de los meses de muestreo, varió notablemente. En el mes de octubre se registraron 358 visitas florales; de éstas, 203 (56,7 \%) fueron de himenópteros, 133 (37,1 \%) de dípteros, $21(5,8 \%)$ de coleópteros, además de una $(0,5 \%)$ visita de lepidóptero. En noviembre se registraron 383 visitas florales; de éstas 280 (73,1\%) fueron coleópteros, 57 $(14,8 \%)$ himenópteros y 46 (12\%) dípteros (figura 1). Entre las especies comunes para ambos meses, solo Bombus $s p$. no presentó más del $1 \%$ de visitas florales.
Entre las especies muestreadas en el mes de octubre, ninguna tuvo una ocurrencia mayor al $1 \%$; sin embargo, las familias Halictidae, Syrphidae y Muscidae, que fueron comunes para ambos meses de muestreo, presentaron una mayor actividad polinizadora en el período de octubre. Para noviembre, las especies que tuvieron una ocurrencia superior a $10 \%$ fueron los coleópteros de la familia Melyridae y Mordella luctuosa (cuadro 3). Estas especies realizaron más del $33 \%$ de las visitas registradas. El coleóptero de la familia Melyridae realizó comparativamente la mayor cantidad de visitas ( $21 \%$ del total observado) lo cual representa $52 \%$ del total de visitas de coleópteros. Sin embargo, éste no fue registrado dentro del ensamble de visitantes en más de la mitad de los nueve días observados.

Relaciones entre número de flores abiertas por planta y la temperatura con la frecuencia de visitantes florales. El análisis de correlación lineal de cada orden de polinizadores con la temperatura ambiental no encontró relaciones significativas en tres de los grupos (Coleoptera, $t=0,3682$; Diptera, $t=0,8987$; Lepidoptera, $t=0,4758$; $P<0,05)$. Para Hymenoptera se presentó una significancia de $t=0,0466 ; P<0,05$. El grado de relación lineal entre el número de flores abiertas por planta con cada orden de insectos, un grupo presentó una actividad significativa (Coleoptera, $t=0,0116 ; P<0,05)$. Los tres restantes ordenes encontrados no presentaron relación significativa (Hymenoptera, $t=0,7554$; Diptera, $t=0,2431$; Lepidoptera, $t=0,7253 ; P<0,05)$ (cuadro 3). 
Cuadro 3. Coeficientes de correlación entre el número de flores abiertas por planta y la temperatura ambiental con cada orden de potenciales polinizadores de E. nitens en el huerto semillero de la hacienda Rucamanqui, Huepil, región del Biobío. $\mathrm{r}=$ coeficiente de correlación de Pearson, rs = coeficiente de correlación de Spearman.

Correlation analysis between the number of open flowers per plant and temperature for each insect floral order $E$. nitens in the clonal seed orchard of farm "Rucamanqui", Huepil, Biobío Region. $\mathrm{r}=$ coefficient of Pearson correlation $\mathrm{t}$, $\mathrm{rs}=$ coefficient of Spearman correlation. $*=P<0.05$, ns = not significant.

\begin{tabular}{lcc}
\hline & Número de flores abiertas por planta & Temperatura ambiental $\left({ }^{\circ} \mathrm{C}\right)$ \\
\hline Número de visitantes de Hymenoptera & $\mathrm{r}=-0,122 \mathrm{~ns}$ & $\mathrm{r}=0,279 *$ \\
Número de visitantes de Coleoptera & $\mathrm{rs}=0,567 *$ & $\mathrm{rs}=-0,183 \mathrm{~ns}$ \\
Número de visitantes de Diptera & $\mathrm{r}=-0,434 \mathrm{~ns}$ & $\mathrm{r}=-0,049 \mathrm{~ns}$ \\
Número de visitantes de Lepidoptera & $\mathrm{rs}=0,137 \mathrm{~ns}$ & $\mathrm{rs}=-0,274 \mathrm{~ns}$ \\
\hline
\end{tabular}

$*=P<0,05 ;$ ns $=$ no significativo.

El análisis microscópico permitió el reconocimiento de siete tipos de polen presentes en el cuerpo de insectos. Del total encontrado, seis pertenecen a los tipos morfológicos de las familias Proteaceae, Onagraceae, Anacardiaceae, Verbenaceae, Rosaceae y Solanaceae. Un tipo de polen fue determinado hasta el nivel taxonómico de especie, E. nitens, que fue observado en el cuerpo de Mordella luctuosa, Corynura sp. y Syrphus reedi (figura 2).

\section{DISCUSIÓN}

La generalización demostrada en este trabajo ha sido considerada por muchos autores, una consecuencia de factores ecológicos, genéticos o filogenéticos que van a limitar el desarrollo de sistemas especializados. Dentro de esta gran gama de visitantes florales, en el mes de octubre destacó la visita de los halíctidos Caenohalictus thauca y C. opacus que mostraron una relación positiva con la temperatura ambiental, a pesar de que el número de flores del huerto fue significativamente menor al mes de noviembre.

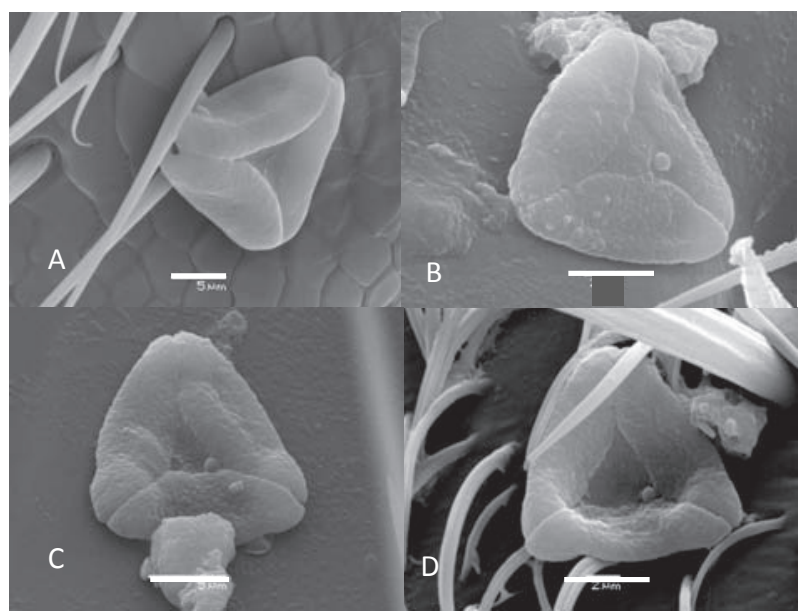

Figura 2. Polen de E. nitens observado en cuerpo de A) Mordella luctuosa, B) y C) Corynura sp. D) y Syrphus reedi. Mediante microscopía electrónica de barrido (barra: $5 \mu \mathrm{m}$ ).

Eucalyptus nitens pollen, found in the body of A) Mordell mournful, B) and C) Corynura sp., and D) Syrphus reedi. By scanning electron microscopy (bar: $5 \mu \mathrm{m}$ ).
Esta evidencia nos permite suponer, para estas abejas halíctidas, que las condiciones térmicas influyen más fuertemente sobre la frecuencia en la actividad de polinizadores, que la disponibilidad del número de flores. La relación positiva con la temperatura ambiental podría deberse a su capacidad de termorregulación. Esta capacidad de termorregulación, decrece a medida que el tamaño corporal disminuye (Bishop y Armbruster 1999). Caenohalictus sp. es un himenóptero de tamaño pequeño $(\sim 3-4 \mathrm{~mm})$, que solo realiza vuelos a cortas distancias (1-3 $\mathrm{m})$ y cuyas poblaciones son muy locales, teniendo probablemente ámbitos de hogar restringidos. Esto indica que las poblaciones de Caenohalictus sp. podrían tener cierta tendencia a asociarse a lugares con microclimas más cálidos. Sin embargo, otros caracteres de las plantas (duración de la floración, magnitud de la oferta simultánea de flores, etc.), además de los considerados en relación directa con los insectos polinizadores, pueden también estar sometidos a presiones de selección con relación al grado de diversificación del ensamble de visitantes florales.

Por otro lado, sería importante examinar las interacciones que se puedan dar con la flora adyacente o rastrera al huerto semillero. Se tienen registros de que la remoción del hábitat remanente que acompaña a un sitio de estudio puede afectar las relaciones mutualistas de una forma compleja. Una buena alternativa sería ampliar la investigación de la carga polínica de los visitantes florales para evaluar la factibilidad de tener una flora adyacente o rastrera, para el incremento del desempeño reproductivo de esta especie exótica. Es importante destacar que la baja frecuencia de visitas de A. mellifera a las flores de E. nitens contrasta con lo ocurrido en Tasmania, donde es frecuente que estos insectos visiten flores de E. nitens de mayor tamaño (Hingston y McQuillan 2000, Hingston et al. 2004), aunque hay registros que muestran que este insecto visita eucaliptos con flores más pequeñas (Griffin et al. 2009, Fernández 2010) como sucede en E. nitens. Dentro de la serie Viminalis, esta especie es la que presenta los elementos florales de menores dimensiones. En el presente estudio, A. mellifera visitó varias inflorescencias ${ }^{2}$. Además tiene registros de ser muy abundante en huertos semilleros de E. globu-

${ }^{2}$ Observación personal de María-José Sanzana. 
lus ${ }^{3}$ y en un huerto de E. nitens de la comuna de Mulchén, Región del Biobío, Chile (Fernández 2010).

En noviembre la visita de coleópteros polinizadores de E. nitens, principalmente de una especie de la familia Melyridae, se incrementó notablemente. Este resultado es coincidente con un estudio en huertos semilleros en Tasmania (Hingston et al. 2004), en donde se encontró una mayor abundancia de Coleoptera en dos de los cinco huertos estudiados. Tanto en el estudio realizado en Tasmania, como en este trabajo, la frecuencia de visitas de este grupo de insectos puede estar determinada por la presencia de vegetación nativa. Especies de Eucalyptus, como E. foecunda Schau., E. cylindrifolia Maiden et Blakely (Hawkeswood 1981) y E. costata (Behr. et F. Muell. Ex F. Muell.) (Horskins y Turner 1999), reciben más visitas de coleópteros que de otro grupo de insectos.

La mayor actividad del coleóptero de la familia Melyridae en el mes con más disponibilidad en el número de flores, puede encontrar su explicación en la biología de los coleópteros. Es probable que este insecto concentre su actividad en sectores que presenten mayor cantidad de recursos florales, lo cual explicaría la ausencia de visitas en el mes de octubre. En este estudio se observó a este pequeño coleóptero copulando sobre las inflorescencias de $E$. nitens. Se ha documentado que el desempeño copulatorio de coleópteros en general, mejora con la cantidad de polen y tejidos nutritivos, además de usar a las flores como lugar de refugio para el apareamiento (Webber y Gottsberger 1993); sin embargo, en el examen del cuerpo de la especie aquí encontrada, no se observaron granos de polen.

En cuanto a los dípteros, según Proctor et al. (1996) afirman que las especies de Muscidae son visitantes florales ocasionales o regulares; sin embargo, Syrphidae es una familia especialista en polinización, ya que sus especies presentan adaptaciones especiales en el labelo, en el número de cerdas del aparato bucal y en las dimensiones de la proboscis, lo que les permitiría alimentarse de néctar o polen. Muscidae y Syrphidae poseen alta movilidad (Amat y Blanco 2003). En este contexto, la alta movilidad y la condición de ectotermia de dípteros, pudieron ser determinantes a la hora de visitar las flores de E. nitens.

Se ha documentado que la existencia de un gradiente de humedad podría estar ejerciendo mayor influencia sobre la estructura de los polinizadores, que la temperatura ambiental. Hawkins et al. (2003) realizan una revisión de estudios de gradientes de riqueza de plantas y animales (que incluyó invertebrados). Este mostró, que en la mayor parte del planeta las variables relacionadas con el agua (e.g. precipitación) fueron mejores predictores de las especies que las variables de "energía" (e.g. temperatura), especialmente en el hemisferio sur. Sin embargo para Apablaza (2000) la humedad es el segundo factor de importancia después de la temperatura que afecta la estructura de los polinizadores. El efecto directo que ejerce la humedad sobre los procesos fisiológicos de los insectos no es tan

\footnotetext{
${ }^{3}$ Observación personal de Jaime Espejo.
}

crítico como el efecto que lleva a cabo la temperatura, pero ambos factores juntos ejercen una notable influencia sobre la biología y la distribución de insectos, al afectar a plantas y animales.

\section{CONCLUSIONES}

La ausencia de especialización en los Eucalyptus spp. es demostrada por la gran gama de insectos visitantes como es el caso de coleópteros, himenópteros, dípteros y lepidópteros. Las diferencias en el ensamble de polinizadores descritas en este trabajo podrían ser determinantes en la producción de semillas de E. nitens, ya que es un hecho que la producción de semilla es debido a la visita de una gran gama de insectos, lo que se estaría revelando en este estudio al encontrar una alta cantidad de vectores en la polinización.

La frecuencia de visita de polinizadores es altamente variable dentro del período de muestreo, principalmente frente a condiciones de temperatura y de densidad de flores abiertas por planta. Para este estudio, estos son factores que pueden generar variación dentro de cada grupo de polinizadores.

\section{AGRADECIMIENTOS}

Los autores agradecen al Proyecto DIUC 210.113.0791.0 de la Dirección de Investigación de la Universidad de Concepción por el apoyo económico de este estudio. A la Dra. Viviane Jerez, Dr. Christian González y MSC Alicia Marticorena, por su ayuda en la determinación de insectos y polen, respectivamente. A Doris Smulders, Verónica Emhart y a la empresa Forestal Mininco S.A, por las facilidades concedidas para desarrollar este trabajo. Por último, a los Sres. Raúl Alarcón, Julio Pugin y Hugo Pacheco, del Laboratorio de Microscopía Electrónica de la Universidad de Concepción, por las ilustraciones de los granos de polen.

\section{REFERENCIAS}

Amat G, E Blanco. 2003. Los Humedales de Bogotá y la Sabana. Tomo I. Bogotá, Colombia. Colombia Ediciones. 90-106 p. Apablaza, GE. Patología de cultivos, epidemiología y control holístico. Santiago, Chile. Ediciones Universidad Católica de Chile. 347 p.

Bishop JA, WS Armbruster. 1999. Thermoregulatory abilities of Alaskan bees: effects of size, phylogeny and ecology. Functional Ecology 13: 711-724.

Brown Jr KS, AVL Freitas. 2002. Diversidade biológica no alto Juruá: avaliação, causas e manutenção. In Carneiro da Cunha MM, MM Almeida (orgs). Enciclopédia da floresta. O alto Juruá: práticas e conhecimentos das populações. Companhia das Letras, São Paulo. p. 33-42.

Fernández A. 2010. Evaluación de la actividad polinizadora de insectos presentes en un huerto semillero clonal de Eucalyptus nitens. Tesis Ingeniero Agrónomo. Temuco, Chile. 
Facultad de Recursos Naturales, Universidad Católica de Temuco. $20 \mathrm{p}$.

Griffin AR, AB Hingston, CP Ohmart. 2009. Pollinators of Eucalyptus regnans (Myrtaceae), the world's tallest flowering plant species. Australian Journal of Botany 57: 18-25.

Hawkins BA, R Field, HV Cornell, DJ Currie, JK Guegan, DM Kaufman, JT Kerr, GG Mittelbach, T Oberdorff, EM O'Brien, EE Porter, JRG Turner. 2003. Energy, water, and broad-scale geographic patterns of species richness. Ecology 84: 3105-3117.

Hingston AB, PB MacQuillan. 2000. Are pollination syndromes useful predictors of floral visitors in Tasmania? Austral Ecology 25: 600-609.

Hingston AB, BM Potts, PB MacQuillan. 2004. Pollination services provided by various size classes of flower visitors of Eucalyptus globulus ssp. globulus (Myrtaceae). Australian Journal of Botany 52(3): 353-369.

INFOR (Instituto Forestal, CL). 2004. Eucalyptus nitens en Chile: Primera Monografía. Valdivia, Chile. CORFO. (Informe Técnico $\mathrm{N}^{\mathrm{o}} 165$ ).

Jordano P, J Bascompte, JM Olesen. 2003. Invariant properties in coevolutionary networks of plant-animal interactions. Ecology Letters 6: 69-81.

Kearns CA, DW Inouye. 1993. Techniques for pollination biologists. University Press of Colorado. $583 \mathrm{p}$.

Lopetegui C. 2002. Antecedentes fonológicos de Eucalyptus nitens (Deane et Maiden) Maiden en el Huerto Semillero Clonal Vista Hermosa. 39 p.

Malo JE, J Baonza. 2002. Are there predictable clines in plantpollinator interactions along altitudinal gradients? The example of Cytisus scoparius (L.) Link in the Sierra de Guadarrama (Central Spain). Diversity and Distributions 8: 365-371.

Morales C, MA Aizen. 2002. Does invasion of exotic plants pro- mote invasion of exotic flower visitors? A case study from the temperate forests of the southern Andes. Biological Invasions 4: 87-100.

Ollerton J, L Cranmer. 2002. Latitudinal trends in plant-pollinator interactions: are tropical plants more specialized? Oikos 98: 340-350.

Potts BM, RC Barbour, AB Hingston, RE Vaillancourt. 2003. Genetic pollution of native eucalypt gene pools - identifying the risks. Australian Journal of Botany 51: 1-25.

Proctor M, P Yeo, A Lack. 1996. The Natural History of Pollination. London, UK. Harper Collins. 479 p.

Rozzi R, MTK Arroyo, JJ Armesto. 1997. Ecological factors affecting gene flow between populations of Anarthrophyllum cumningii (Papilionaceae) growing on equatorial- and polar-facing slopes in the Andes of central Chile. Plant Ecology 132: 171-179.

Seastedt TR, DA Jr Crossley. 1984. The Influence of Arthropods on Ecosystems. BioScience 34(3): 157-161.

Speight MR, M Hunter, A Watt. 1999. Ecology of Insects: Concepts and Applications. Editorial Offices. 351 p.

Torres-Díaz C, L Cavieres, C Muñoz-Ramírez, M Arroyo. 2007. Consecuencias de las variaciones microclimáticas sobre la visita de insectos polinizadores en dos especies de Chaetanthera (Asteraceae) en los Andes de Chile central. Revista Chilena de Historia Natural 80: 455-468.

Tscharntke T, R. Brandl. 2004. Plant-insect interactions in fragmented landscapes. Annual Review of Entomology 49: 405-430.

Utelli AB, BA Roy. 2000. Pollinator abundance and behaviour on Aconitum lycoctonum (Ranunculaceae): an analysis of the quantity and quality components of pollination. Oikos 89: 461-470.

Webber AC, G Gottsberger. 1993. Floral biology and pollination of Cymbopetalum euneurum (Annonaceae) in Manaus, Amazonia. Annonaceae Newsletter 9: 25-28. 
